\title{
Perception of Brightness and Brightness Illusions in the Macaque Monkey
}

\author{
Xin Huang, Sean P. MacEvoy, and Michael A. Paradiso \\ Department of Neuroscience, Brown University, Providence, Rhode Island 02912
}

Recent physiological studies show that neural responses correlated with the perception of brightness are found in cortical area V1 but not earlier in the visual pathway (Kayama et al., 1979; Reid and Shapley, 1989; Squatrito et al., 1990; Komatsu et al., 1996; Rossi et al., 1996; MacEvoy et al., 1998; Rossi and Paradiso, 1999; Hung et al., 2001; Kinoshita and Komatsu, 2001; MacEvoy and Paradiso, 2001). However, these studies are based on comparisons of neural responses in animals with brightness perception in humans. Very little is known about the perception of brightness in animals typically used in physiological experiments. In this study, we quantify brightness discrimination, brightness induction, and White's effect in macaque monkeys. The results show that, qualitatively and quantitatively, the perception of brightness in macaques and humans is quite similar. This similarity may be an indication of common underlying neural computations in the two species.

Key words: brightness; brightness perception; color perception; surface perception; macaque; visual illusion
For the purpose of understanding the neural basis of human visual perception, the best widely used animal model is the macaque monkey. What has been learned about the macaque suggests that both its visual physiology and perception are quite similar to that of humans. However, there are large gaps in our understanding of the visual capabilities of this animal that limit our ability to relate the results of neural recordings to visual perception.

Our own studies of the neural representation of brightness information (Rossi et al., 1996; MacEvoy et al., 1998; Rossi and Paradiso, 1999; MacEvoy and Paradiso, 2001) prompted us to study brightness perception in the macaque. There are previous studies of brightness perception in monkeys going back to the early 20th century (Crawford, 1935; Ash, 1951; Davis et al., 1965; Brooks, 1966; Schilder et al., 1971), but these are of limited utility for several reasons. First, some previous studies used diverse types of monkeys other than macaques. Second, almost all of the previous studies were limited to simple discrimination; visual illusions involving brightness were generally not explored. The study of visual illusions is especially valuable for comparisons with physiological results because one can test whether neural responses correlate with the physical or perceptual properties of objects.

The third limitation of previous studies is that they often did not lead to the construction of psychometric functions as is typical in human psychophysics experiments. In relating physiology to psychophysics, psychometric functions are of great value because they can be compared with "neurometric" functions to assess whether neurons in a particular brain area carry informa-

Received April 22, 2002; revised Aug. 20, 2002; accepted Aug. 22, 2002.

This research was supported by the United States National Eye Institute. We thank Lisa Kinsella for technical assistance and Drs. Rick Born, Ken Britten, Greg DeAngelis, Jennifer Groh, Nikos Logothetis, Earl Miller, and Michael Shadlen for valuable technical advice.

Correspondence should be addressed to Michael A. Paradiso, Department of Neuroscience, 192 Thayer Street, Brown University, Providence, RI 02912. Email: michael_paradiso@brown.edu.

Copyright ( 2002 Society for Neuroscience 0270-6474/02/229618-08\$15.00/0 tion comparable with that used by an animal in the performance of a visual task.

With the limitations of previous animal perception experiments in mind, we set out to explore brightness perception in the macaque. Animals were trained to make discriminations in three situations: simple brightness discrimination of isolated gray patches, simultaneous brightness contrast (brightness induction), and White's effect.

\section{MATERIALS AND METHODS}

General procedures. Two adult rhesus monkeys (Macaca mulatta) weighing $5.2 \mathrm{~kg}$ (monkey PN) and $4.8 \mathrm{~kg}$ (monkey HN) served as subjects in these experiments. Before behavioral training, aseptic surgery with isoflurane anesthesia was performed to attach a head post and place a scleral search coil on the right eye (Judge et al., 1980). All procedures conformed to National Institutes of Health guidelines and were approved by the Brown University animal care and use committee.

During training and experimental sessions, animals sat in a primate chair facing a computer display $(640 \times 480$ pixel resolution $)$ at a distance of $93 \mathrm{~cm}$. At the beginning of each session, animals were adapted to the darkened room for $10 \mathrm{~min}$. Head position was fixed, and eye position was monitored using the scleral search coil or, in some cases with animal HN, an ISCAN (Burlington, MA) infrared eye tracking system.

Brightness discrimination. Monkeys were trained on a two-alternative forced-choice task to make saccadic eye movements to the brighter of two simultaneously presented gray disks. Each disk had a radius of $1.0^{\circ}$, and the centers of the two disks were separated by $6.0^{\circ}$.

Each trial began with the appearance of a small fixation cross centered on the display (Fig. $1 A$ ). Animals were required to fixate within a $1.8^{\circ}$ diameter window for $1 \mathrm{sec}$, after which the two disks appeared. The animals had to wait until the fixation cross was extinguished (a random interval between 1.2 and $1.8 \mathrm{sec}$ ) and then, within $300 \mathrm{msec}$ of fixation cross offset, saccade to the disk that was brighter. Animals received a liquid reward for correctly choosing the brighter patch, but only after maintaining fixation on it for $700 \mathrm{msec}$ (Fig. $1 B$ ). Trials were aborted and no reward was given if the animals broke fixation at any time while the cross was present, if they attempted to "change" their decision by glancing to one disk and then the other, or if they moved their eyes to any portion of the display other than either disk after the fixation cross disappeared. To negate any bias that the monkeys might have for saccades to one side or the other, the brighter patch randomly alternated between the right and left positions (any left-right differences in monitor output were corrected by adjusting lookup table values).

When performance stabilized after several weeks of training, bright- 
A Brightness Discrimination

C Brightness Induction

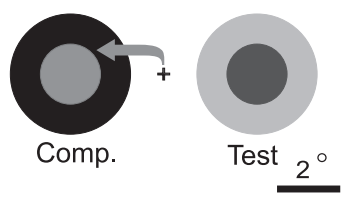

D White's Effect

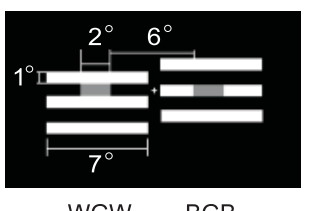

WGW

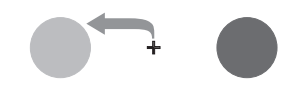

B Paradigm for Discrimination \& Induction

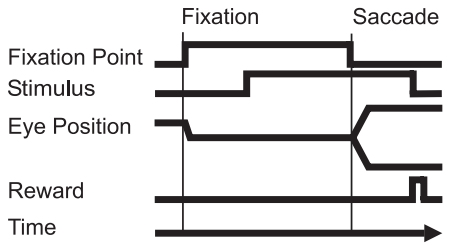

$1 \mathrm{Sec}$.

E Paradigm for White's Effect Free Viewing Fixation Saccade

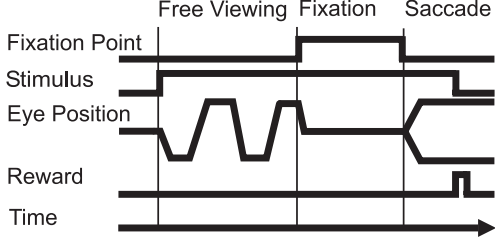

$1 \mathrm{Sec}$.
Figure 1. Experimental design. $A$, In brightness discrimination, an animal fixated a central point and then saccaded to the brighter of two $1^{\circ}$ radius disks centered at $3^{\circ}$ eccentricity to each side of fixation. $B$, In both discrimination and induction experiments, animals fixated for $1 \mathrm{sec}$, two stimuli were presented, and, after a variable delay of 1.2-1.8 sec, the fixation point was extinguished, cueing the animal to saccade to the brighter target. $C$, The brightness induction task was identical to brightness discrimination, except that the two disks were surrounded by $1^{\circ}$ thick annuli. The test disk had a luminance of either $12.6 \mathrm{or} 32.2 \mathrm{~cd} / \mathrm{m}^{2}$, and the test surround was given six to seven different luminances. The comparison disk took on a range of luminances, and the comparison surround was fixed at $1.3 \mathrm{~cd} / \mathrm{m}^{2} . D$, To study White's effect, two square-wave luminance gratings were placed side-by-side. The black and white stripes had luminances of 0.2 and $81 \mathrm{~cd} / \mathrm{m}^{2}$, respectively. A $1^{\circ} \times 2^{\circ}$ gray patch was superimposed on a black stripe on one of the gratings and a white stripe on the other. The patch on the black stripe (white-gray-white or WGW stimulus) had a luminance of 14.1 or $31.3 \mathrm{~cd} / \mathrm{m}^{2}$, and the luminance of the patch on the white stripe (black-gray-black or BGB stimulus) was variable. The animal saccaded to the patch that appeared brighter. $E$, In the White's effect experiment, animals were allowed to freely view both stimuli. After $2-3 \mathrm{sec}$ of free viewing, a fixation point appeared, and the animals had to fixate this $\left(1.8^{\circ}\right.$ diameter window) for a variable interval of $1.2-1.8 \mathrm{sec}$. The animals saccaded to the brighter of the two gray patches when the fixation point was extinguished.

ness judgments were recorded. On data collection days, monkeys completed 40-100 warm-up trials before behavioral responses were recorded.

Brightness induction. The task in the brightness induction experiment was nearly identical to that described for simple discrimination. The stimulus differed in that each gray disk was surrounded by a $1.0^{\circ}$ thick annulus (Fig. 1C). To human observers, a gray disk surrounded by a white annulus appears darker than a gray disk surrounded by black. In each block of trials, the luminance values of one disk and its annular surround (termed the "test" disk and surround) were held constant. The test disk was fixed at 12.6 or $32.2 \mathrm{~cd} / \mathrm{m}^{2}$, and, across blocks, the test surround had six or seven different values. In each trial, the luminance of the other disk (termed the "comparison disk") was chosen from a set of values ranging from far below to far above that of the test disk. The luminance of the second annular surround (the "comparison" surround) was always 1.3 $\mathrm{cd} / \mathrm{m}^{2}$. In human psychophysics experiments, subjects would typically use the method of adjustment to set the comparison disk to match the test disk. With the monkeys, it was necessary to record discriminations with a range of comparison stimuli to quantify the brightness of the test disk, because the monkeys' task was simply to saccade to the brighter disk. The timing of stimulus presentation and behavioral response was identical to that used in the brightness discrimination experiments described above.

Training the animals to perform the induction experiment required great care, because the addition of the surrounds had, at the outset, an unknown impact on the animals' perception of brightness. One approach would have been to train the animals extensively on the simple brightness discrimination task in the absence of surrounds and then add the surrounds and immediately record responses while rewarding randomly. We rejected this approach because of concerns that the animals might start guessing or base their responses on the annuli rather than the disks. Instead, we trained the animals with the surrounds present but used a reduced set of stimulus conditions in which the only possible effect of the surrounds was to exaggerate a luminance-based difference in brightness between the test and comparison disks. For example, an animal would be shown one stimulus with a dark surround and bright center along with a second stimulus having a bright surround and dark center. The animal was rewarded for choosing the more luminous center without fear of introducing a bias, because a judgment based on luminance or brightness would be the same. This approach involved the minimal assumption that the annular surround had either no effect or an inhibitory effect on the brightness of the disk but not a facilitatory effect (i.e., the surround influence is not opposite in sign for monkeys and humans). This strategy did pose the risk of training the animals to pick the side with the darker surround rather than the lighter center. To counter this, we included control conditions in which the monkey was rewarded for choosing an extremely bright center with a bright annulus over an almost black center with a dark annulus.

In the actual experiments, a wider range of center and surround luminance values was used, and we used a probabilistic reward scheme to avoid introducing a bias into the monkeys' judgments. In cases in which decisions based on luminance or brightness would unequivocally be the same, the animal was rewarded $100 \%$ for choosing the more luminous patch, as during training. In all other cases, meaning in those conditions that would make up the critical portion of the psychometric curve, the animals were randomly rewarded on $50 \%$ of the trials. To ensure that this reward scheme did not encourage random guessing, we continued to interleave conditions which verified that the monkey was basing its judgments on the brightness of the disks and not the surrounds. Overall, assuming correct performance on control trials, the monkeys received juice on $75 \%$ of trials, on average.

At least 100 trials were recorded with each test surround luminance value, and the induction experiment was repeated with six or seven different values. Before data collection, animals completed 65-145 warm-up trials.

White's effect. The stimulus pairs used to explore White's effect closely resembled those used in human psychophysical experiments. They consisted of two $0.5 \mathrm{cycle} /{ }^{\circ}$ square-wave luminance gratings side-by-side. On one of the gratings, a rectangular gray patch was substituted for a portion of a black stripe, and, on the other grating, a gray patch replaced part of a white stripe (Fig. $1 D$ ). The perceptual illusion seen by humans is that the gray patch with its long sides surrounded by black appears darker than the other patch with long sides surrounded by white. Thus, the illusion seems opposite that of brightness induction in which a dark surround causes a gray patch to appear lighter rather than darker.

The animals were trained to saccade to the brighter of the gray patches, either the one with long black flanks or the other surrounded by long white flanks. For brevity, we will refer to the stimulus in which a gray patch is flanked top-and-bottom by long black bands as BGB (blackgray-black) and the stimulus with gray flanked by white as WGW (white-gray-white). The luminances of the black and white flanks were fixed at 0.2 and $81 \mathrm{~cd} / \mathrm{m}^{2}$, respectively. To study White's effect, brightness discriminations were made with the WGW gray patch fixed at either 14.1 or $31.3 \mathrm{~cd} / \mathrm{m}^{2}$ while the luminance of the $\mathrm{BGB}$ patch was varied.

Our initial experiments were conducted with a fixation paradigm similar to that used in the discrimination and induction experiments. However, it appeared that the complexity of the stimulus and the peripheral placement of the gray patches made the task difficult for the monkeys. Therefore, we changed to a free-viewing paradigm that gave more reliable results by allowing the animals to look back and forth between the stimuli before rendering a decision. This is the approach used in comparable human psychophysics experiments.

Eye position traces observed during data collection indicated that the free viewing period was used by the monkeys to examine the gray patches. After 2-3 sec of free viewing, a fixation cross appeared that the animals had to foveate within a $1.8^{\circ}$ diameter window. After holding fixation for a variable interval $(1.2-1.8 \mathrm{sec})$, the fixation cross was turned off, allowing the animals to saccade to the gray patch that was perceived brighter.

Training and reinforcement were managed in a similar manner to the brightness induction task. Once again, we assumed that the surrounding stimuli had either no effect on the brightness of the gray patches or an 
A

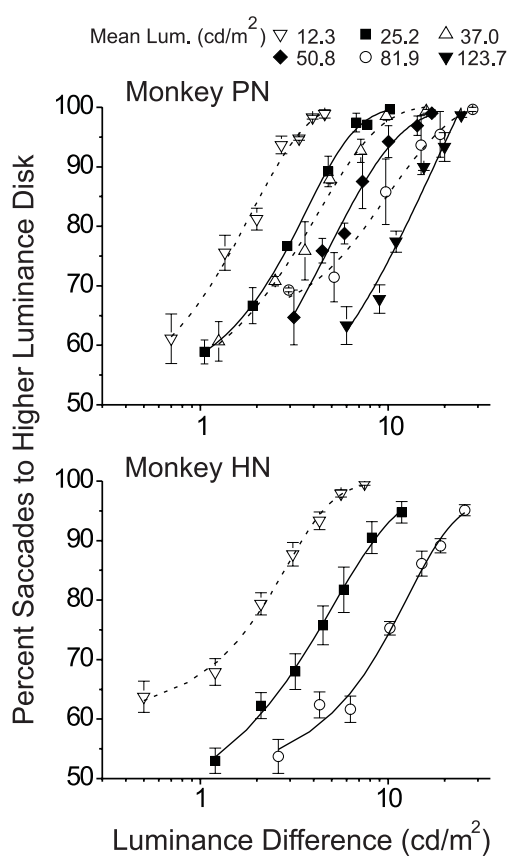

B

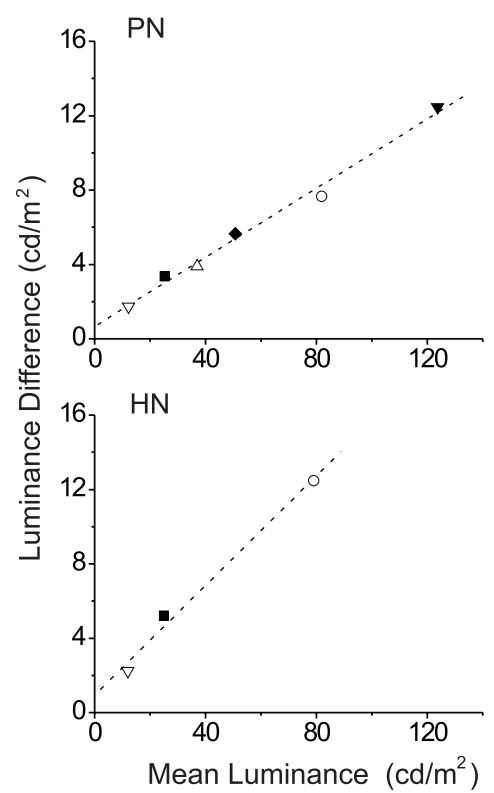

Figure 2. Brightness discrimination in two animals (PN and HN). $A$, The percentage of saccades to the brighter, more luminous, disk as a function of the luminance difference between disks. Different symbols indicate data collected at various mean luminance values. $B$, The luminance difference required to achieve $80 \%$ correct on the discrimination was linearly related to mean luminance (linear fit indicated by dashed line). The Weber fraction was 0.11 for animal PN and 0.18 for animal $\mathrm{HN}$. consisted of test and comparison disks, each with an annular surround. The test disk luminance was fixed at 12.6 or $32.2 \mathrm{~cd} / \mathrm{m}^{2}$, and the comparison surround was fixed at $1.3 \mathrm{~cd} / \mathrm{m}^{2}$. To quantify brightness induction, the luminance of the comparison patch was varied with the test surround fixed at one of six to seven different luminance levels in different blocks of trials (indicated by different symbols in Fig. $3 A$ ).

Each curve in Figure $3 A$ shows the percentage of saccades that the animal made to the comparison disk as a function of the difference in luminance between the comparison and test disks. For all data points to the left of the vertical line, the test disk was more luminous than the comparison disk, and, for all to the right, it was less luminous. The horizontal line marks 50\% performance; any data point in the space above the line indicates that the monkey perceived the comparison disk to be brighter than the test disk, and, for any point below the line, the monkey perceived the comparison disk to be darker than the test disk. If there were no effect of the annular surrounds, every curve should pass through the $50 \%$ level of performance when there was no luminance difference between test and comparison patches. All of the data in Figure $3 A$ come from experiments with a test disk luminance of $32.2 \mathrm{~cd} / \mathrm{m}^{2}$.

We fit sigmoidal functions to the set of responses obtained with each test surround luminance, and we took the comparison luminance in which each curve crossed the horizontal line at $50 \%$ as the theoretical brightness match for the test disk with that surround intensity. For example, with animal PN (top panel), the curve drawn through open squares represents the psychometric function obtained when the test and comparison surrounds were of equal luminance. The curve crosses $50 \%$ performance when the comparison disk had the same luminance as the test disk, consistent with the fact that this special case was simple brightness discrimination. As test surround luminance increases (different symbols in the figure), the curves cross the $50 \%$ performance line when the comparison disk luminance was progressively lower than the fixed test disk luminance. In other words, test surrounds of higher luminance diminished the apparent brightness of the test patch two monkeys in a stimulus situation that produces perceptual brightness induction in humans. The stimuli in this experiment for monkey $\mathrm{HN}$.

\section{Brightness induction}

Figure 3 shows data from brightness discriminations made by the 
A

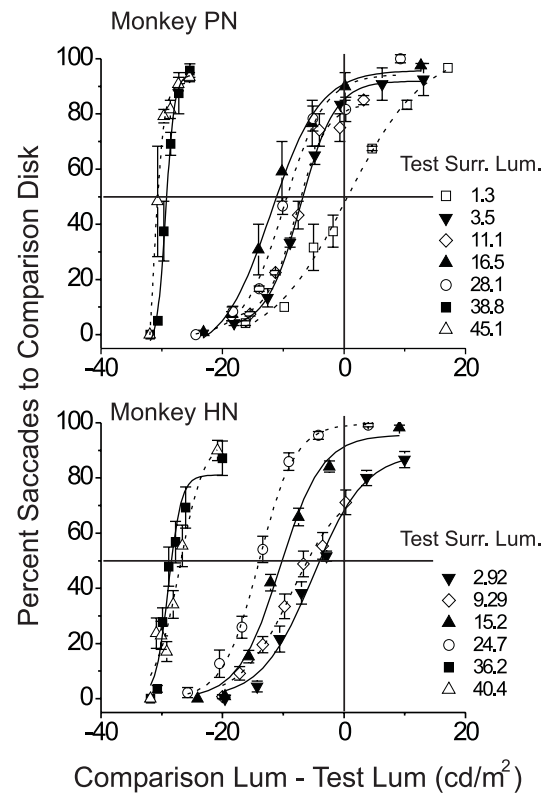

B

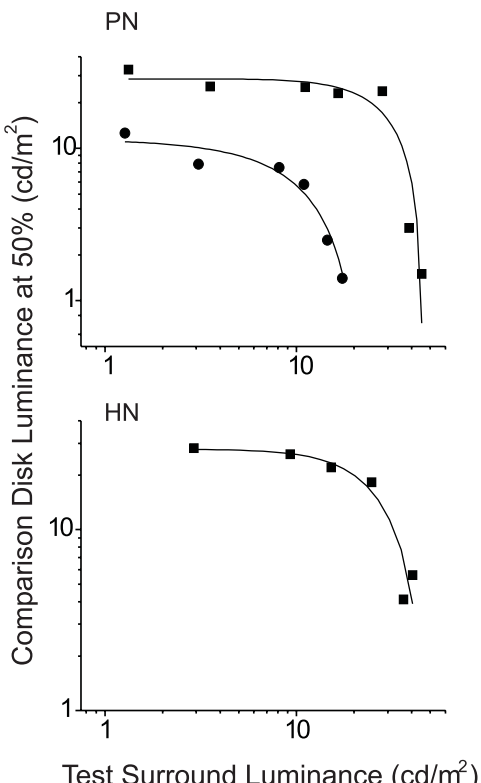

Figure 3. Brightness induction in two animals. Animals saccaded to the test or comparison disk to indicate which appeared brighter. $A$, Test disk luminance was $32.2 \mathrm{~cd} / \mathrm{m}^{2}$, test surround luminance was fixed at the level indicated by the various symbols, and comparison surround luminance was $1.3 \mathrm{~cd} / \mathrm{m}^{2}$. As the comparison disk luminance changed, the difference between comparison and test disk luminances varied (abscissa). As this difference became more positive, more saccades were made to the comparison disk, consistent with it appearing brighter. Lines drawn through the symbols are sigmoidal fits. $B$, The intersection between fitted curves in $A$ and the horizontal line at $50 \%$ was used to determine the comparison disk luminance at which the brightness of the comparison disk appeared to match the brightness of the test disk. The matching comparison disk luminances are plotted as a function of the test surround luminance. For both animals, data are shown for the $32.2 \mathrm{~cd} / \mathrm{m}^{2}$ test disk luminance used in $A$ (squares), and additional data with a test disk at $12.6 \mathrm{~cd} / \mathrm{m}^{2}$ are shown for animal PN (circles). (i.e., brightness induction). Very similar results were obtained with the second monkey (Fig. $3 A$, bottom panel).

Figure $3 B$ shows the brightness matching points (obtained from the sigmoidal fits) plotted against test surround luminance. This manner of data presentation is more typical of human psychophysical experiments in which the method of adjustment is used. For monkey PN (top panel), the two curves correspond to results obtained with two different test disk luminances (32.2 and 12.6 $\mathrm{cd} / \mathrm{m}^{2}$ ); monkey HN (bottom) was tested with a single test disk luminance $\left(32.2 \mathrm{~cd} / \mathrm{m}^{2}\right)$. These curves confirm the trend evident in the raw data: higher values of test surround luminance elicit lower estimates of test disk brightness. Of particular note is the rapid change in the brightness matching points from test surround luminance 28.1 to $38.8 \mathrm{~cd} / \mathrm{m}^{2}$ on the square-symbol curves in Figure $3 B$. These two test surround luminances span the test patch luminance of $32.2 \mathrm{~cd} / \mathrm{m}^{2}$ and thus correspond to a switch from the patch being an increment to a decrement.

Although these data strongly suggest that monkeys perceive a disk on a dark background to be brighter than one on a bright background, the data were collected in a different manner than typical side-by-side comparisons in human induction experiments. As an additional test of the animals' perception of induction, for one monkey (PN), we included conditions that allowed a side-by-side comparison of stimuli. In other words, the monkey simultaneously saw two identical disks with different surround luminances as a human would in a typical experiment. We used surrounds only from neighboring points in Figure $3 B$ (i.e., the surround luminance from the first data point vs the second point, the second point vs third, etc). We found that, across the six possible test surround comparisons from the top curve in Figure $3 B$ (test disk, $32.2 \mathrm{~cd} / \mathrm{m}^{2}$ ), $91 \%$ of the time monkey PN judged the disk with the less luminous surround to be brighter. From the five possible comparisons in the bottom curve of Figure $3 B$ (test disk, $\left.12.6 \mathrm{~cd} / \mathrm{m}^{2}\right), 88 \%$ of the time the disk with the less luminous surround was judged to be brighter. Thus, these data collected with a paradigm identical to that used in human experiments show judgments consistent with the perception of brightness induction.

\section{White's effect}

Figure 4 shows psychometric functions derived from brightness judgments using a White's effect stimulus. As in the discrimination and induction experiments, animals were trained to saccade to the brighter of two stimuli. Each data point represents the percentage of saccades made to the gray patch flanked by black bars (BGB patch) as a function of the difference in luminance between the BGB gray patch and the WGW gray patch. The vertical line indicates the condition in which the two gray patches had the same luminance. If there were no brightness illusion (and no inherent response bias), the animals should make $\sim 50 \%$ of their saccades to the BGB side when the gray patches are equally luminous. When the $2^{\circ}$ long gray patches had $7^{\circ}$ long black and white flanks (open symbols), a situation that evokes White's effect in humans, this was clearly not the case: saccades were not made equally to the two gray patches until the BGB gray patch luminance was $18.6 \mathrm{~cd} / \mathrm{m}^{2}$ higher than the WGW patch (for animal $\mathrm{PN}$ ) and $37.3 \mathrm{~cd} / \mathrm{m}^{2}$ higher for animal HN. We cannot tell to what extent this deviation represents a perceptual lightening of the WGW patch versus a darkening of the BGB patch. However, it is clear that, for monkeys, like humans, the patch with white flanks appears brighter than one with dark flanks. The fact that the percentage of saccades to the BGB patch changed systematically as its luminance was varied indicates that the animals were using the patch itself rather than some other configuration cue or flank intensity to make their decisions.

Conceivably, the shift of the curves to the right of the vertical lines could be a result of response bias rather than a perceptual illusion. We examined this possibility by repeating the experiment with the black and white flanks reduced to $2^{\circ}$ in length, matching the length of the gray patches (Fig. 4, filled data points). To humans, this is a situation that evokes brightness induction rather than White's effect. Consistent with the sign reversal in human perception, the curves shift from right of the vertical lines to the left of the vertical lines in Figure 4. To achieve equal numbers of saccades to the two gray patches, the BGB gray patch had to be $10.7 \mathrm{~cd} / \mathrm{m}^{2}$ lower than the WGW patch for animal PN and 5.7 
A
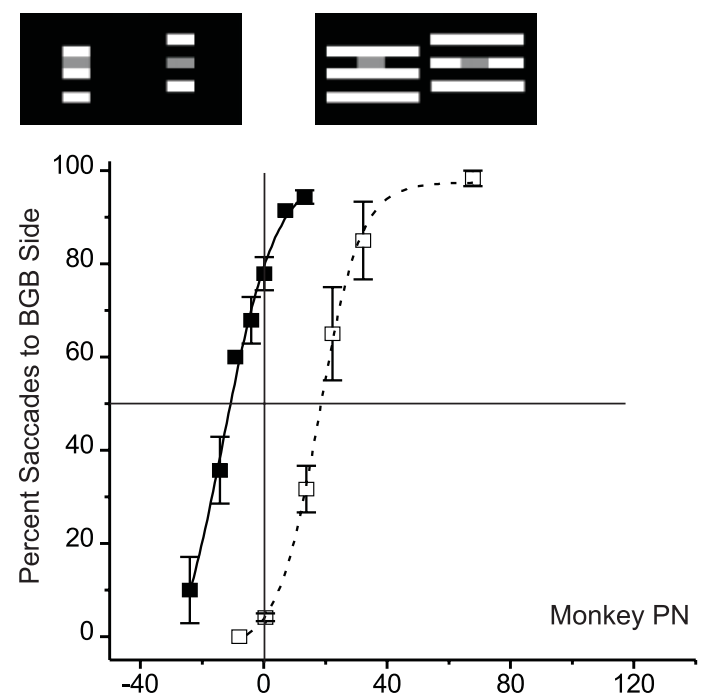

B

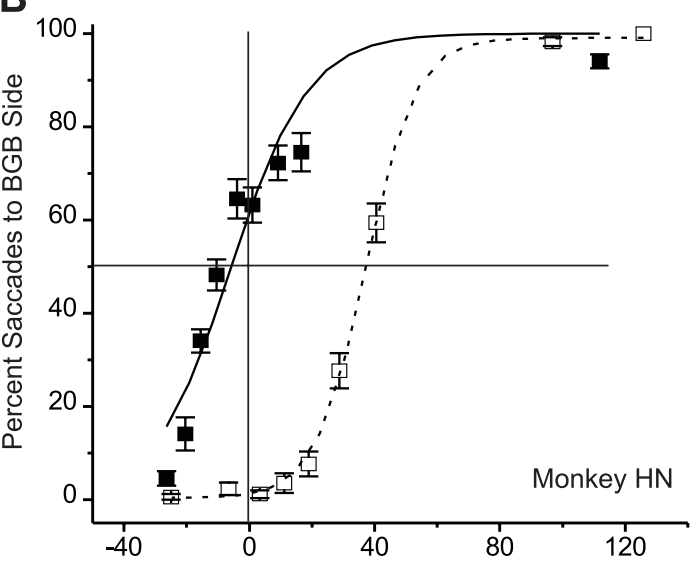

Luminance Difference between Gray Patches $\left(\mathrm{cd} / \mathrm{m}^{2}\right)$ (BGB - WGW)

Figure 4. White's effect in two animals. Animals saccaded to the gray patch on either the BGB or WGW side to indicate which patch appeared brighter. The WGW gray patch was fixed at $31.3 \mathrm{~cd} / \mathrm{m}^{2}$, and the BGB patch luminance was varied. The percentage of saccades to the gray patch on the BGB stimulus was measured as the difference in luminance between the BGB and WGW gray patches changed. The brightness of the two patches was taken to be equal when behavioral performance was 50\% (horizontal lines). When $7^{\circ}$ long flanks were adjacent to the $2^{\circ}$ long gray patches (open symbols and dashed sigmoidal fit curve), 50\% behavioral performance was achieved when the BGB gray patch had a significantly higher luminance than the WGW patch (shift of dashed lines to the right of vertical line). The influence of the flanking lines was dramatically different when the flanks were reduced to $2^{\circ}$ in length, matching the gray patch length ( filled symbols and solid sigmoidal fit curve). The gray patches appeared to be matched in brightness when the BGB patch had a lower rather than higher luminance compared with the WGW patch.

$\mathrm{cd} / \mathrm{m}^{2}$ lower for animal HN. Thus, with a simple manipulation of the stimulus that reverses the perceptual effect in humans, the judgments of the monkeys also reversed. This finding strongly suggests that monkeys perceive White's effect in addition to brightness induction.

The data discussed above show that the monkeys perceived White's effect when the WGW patch luminance was $31.3 \mathrm{~cd} / \mathrm{m}^{2}$. To test the generality of this finding, we also collected data in one monkey with a WGW patch luminance of $14.1 \mathrm{~cd} / \mathrm{m}^{2}$. With no
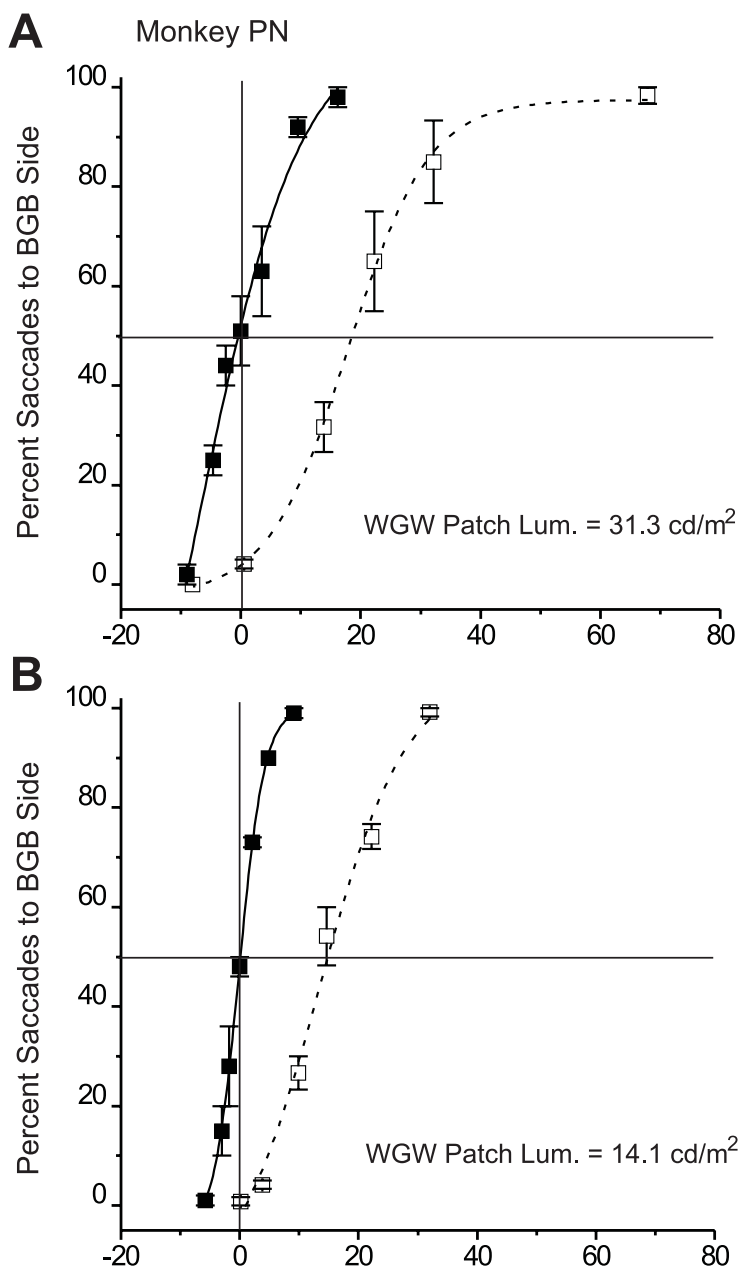

Luminance Difference between Gray Patches $\left(\mathrm{cd} / \mathrm{m}^{2}\right)$ (BGB - WGW)

Figure 5. White's effect in animal PN with two different WGW patch luminances. $A$, Data represented by the open symbols and dashed sigmoidal fit come from experiments with $7^{\circ}$ long flanks and a WGW gray patch luminance of $31.3 \mathrm{~cd} / \mathrm{m}^{2}$. The filled symbols and solid sigmoidal fit were obtained in experiments without flanks. In this latter situation, the curve crosses the $50 \%$ level when the gray patches have identical luminance, consistent with the task being simple brightness discrimination when there are no flanks. $B$, Data represented by the open symbols and dashed sigmoidal fit come from experiments with $7^{\circ}$ long flanks and a WGW gray patch luminance of $14.1 \mathrm{~cd} / \mathrm{m}^{2}$. The filled symbols and solid sigmoidal fit again show simple brightness discrimination in the absence of flanks.

previous experience with this lower luminance gray patch, the monkey immediately made responses consistent with White's effect (Fig. 5B). This is shown by the displacement of the dashed curves to the right of the vertical lines. With the WGW gray test patch at $14.1 \mathrm{~cd} / \mathrm{m}^{2}$ (Fig. $5 B$ ), the BGB gray patch had to be 14.9 $\mathrm{cd} / \mathrm{m}^{2}$ higher for the animal to make equal numbers of saccades to both patches. As already noted, with the gray test patch at 31.3 $\mathrm{cd} / \mathrm{m}^{2}$, the BGB gray patch had to be $18.6 \mathrm{~cd} / \mathrm{m}^{2}$ higher. For comparison, the solid lines in Figure 5 show brightness discriminations made with no flanks.

We conducted a number of additional experiments to confirm and extend the basic findings. In demonstrations of White's effect in humans, unlike the monkey experiments reported so far here, the two gray patches typically have the same luminance. Although we needed to vary the patch luminances to quantify 
A

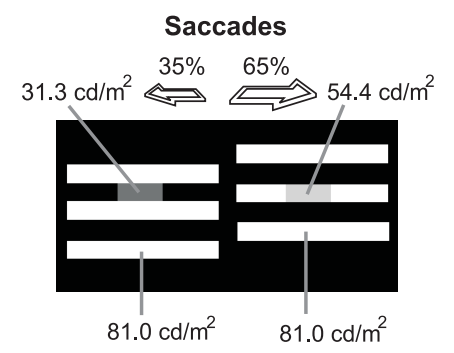

D

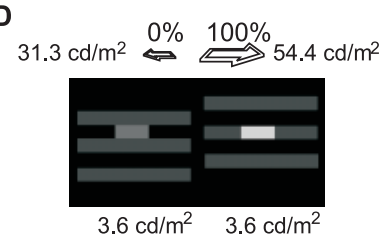

Figure 6. The role of induction in White's effect. $A$, In this condition from the White's effect experiment, the WGW gray patch had a luminance of $31.3 \mathrm{~cd} / \mathrm{m}^{2}$, and the BGB patch had a luminance of $54.4 \mathrm{~cd} / \mathrm{m}^{2}$. The black stripes were $0.2 \mathrm{~cd} / \mathrm{m}^{2}$, and the white stripes were $81.0 \mathrm{~cd} / \mathrm{m}^{2}$ on both sides of the stimulus. With these luminance values, the animal saccaded $65 \%$ of the time to the BGB gray patch, indicating that it appeared brighter. $B$, The stimulus in this condition is identical to that in $A$, except that the white bands on the BGB side have been reduced to 3.6 $\mathrm{cd} / \mathrm{m}^{2}$. With this change, the animal saccaded $100 \%$ of the time to the BGB side, indicating that the gray patch on that side appeared brighter than in $A$. $C$, This stimulus was modified from $A$ by reducing the luminance of the flanking stripes on the WGW side to $3.6 \mathrm{~cd} / \mathrm{m}^{2}$. With this stimulus change, the animal saccaded almost equal numbers of times to the WGW and BGB gray patches. $D$, When the flanks on the WGW stimulus as well as the collinear bands on the BGB stimulus are darker than the gray patches, animals always saccade to the more luminous patch.

White's effect in monkeys, we confirmed our results with a configuration identical to that used in human demonstrations. The BGB and WGW gray patches were given identical luminances of $8.5,14.1,21.7$, or $31.3 \mathrm{~cd} / \mathrm{m}^{2}$, values that all evoke White's effect in humans. Consistent with the human results, animal PN made $100,99,91$, and $96 \%$ of her saccades to the WGW gray patch, indicating that it appeared brighter, although it actually had the same luminance as the BGB patch.

The results shown in Figure 6 suggest part of the mechanism responsible for White's effect. The difference between the stimuli in Figure $6 A$ and $6 B$ is that the band collinear with the gray patch (and the other light bands on this stimulus) on the BGB side is $81.0 \mathrm{~cd} / \mathrm{m}^{2}$ in Figure $6 A$ but $3.6 \mathrm{~cd} / \mathrm{m}^{2}$ in Figure $6 B$. To humans, the perceptual effect of this difference is that, with the same luminance, the BGB gray patch appears lighter in Figure $6 B$ in which the bands collinear with the gray patch are darker (Spehar et al., 1995). As the figure indicates, the monkey also made a much higher percentage of saccades $(100 \%)$ to the BGB gray patch with the stimulus in Figure $6 B$ than in Figure $6 A(65 \%)$. Thus, for both monkeys and humans, darkening the bands collinear with the gray patch makes the gray patch appear relatively brighter. In other words, the collinear flanks produce an induction effect.

Comparing Figure $6 A$ with $6 C$, the difference is that the WGW flanks in Figure $6 A$ have a luminance of $81.0 \mathrm{~cd} / \mathrm{m}^{2}$ and, in Figure
$6 C$, they have a luminance of $3.6 \mathrm{~cd} / \mathrm{m}^{2}$. Note that this is the same luminance difference as between Figure $6 A$ and $6 B$, except here the flanks on the WGW side were changed rather than the bands collinear with the gray patch on the BGB side. The human perceptual effect of darkening the WGW flanks is to make the WGW gray patch lighter. Consistently, in the monkey tested, darker WGW flanks led to an increase in the percentage of saccades to the WGW gray patch (Fig. 6, from 35\% in $A$ to $47 \%$ in $C$ ). Thus, an induction effect is produced by the long flanks as well as the collinear bands.

In summary, in both comparisons (Fig. 6, compare $A$ with $B$ and $A$ with $C$ ), it appears that induction effects are present. However, these influences push the brightness of the test patch in opposite directions. The critical observation that distinguishes the effects of collinear and flanking bands is that the change in the animal's decisions was far smaller when the same luminance difference occurred in the flanks than in the collinear bands. Stated another way, White's effect appears to be based on antagonistic induction processes, but the induction from collinear bands is significantly more potent than induction from flanking areas. For this reason, the overall effect appears to be the opposite of induction if one focuses only on the contrast of the long flanking borders with the gray patches.

In Figure $6 D$, both the flanks on the WGW side and the collinear bands on the BGB side are darker than the gray patches. In this situation, the monkey saccaded $100 \%$ of the time to the more luminous gray patch. This is consistent with the finding in humans that White's effect disappears if the test patch has a luminance higher than the lighter bands in the square wave (Spehar et al., 1995).

\section{DISCUSSION}

The results of this study show a remarkable qualitative and quantitative similarity between brightness perception in humans and macaques. The discussion below addresses the implications of this finding for human-macaque comparisons and brain mechanisms underlying perception.

\section{Did the animals base their decisions on brightness?}

Animals in these experiments clearly used the intensity of visual stimuli to make judgments, but was brightness the quality that they assessed? In the brightness discrimination experiments, brightness covaried with luminance, so the results might validly be called luminance discriminations. It is in the induction and White's effect experiments that the perceptual quality of brightness is distinguished from the physical measure of luminance. The most straightforward evidence that brightness was used is the shifts in the discrimination curves away from simple luminance discrimination.

In the induction experiments, if the animals based their judgments on the luminance of the central disks, they should have made saccades to the comparison and test disks $50 \%$ of the time when the disk luminances were identical. Instead, the curves shifted in a direction that would be called induction in humans. However, might the animals have used something other than the disk brightness, for example, the luminance of the annuli? Several observations argue that both monkeys made their judgments based on the brightness of the disks and not on the luminance of the surrounds. First, in Figure 3A, each curve was constructed from trials in which both test and comparison surrounds had fixed luminance. The perceptual judgments varied systematically and correlated with the brightness of the comparison disk as judged 
by humans. The judgments did not correlate with either surround luminance or test disk luminance. Additionally, on trials in which the test and comparison disks were presented in the absence of surrounds, as well as when their surrounds had identical luminance, both monkeys correctly made saccades to the more luminous center on $\sim 99 \%$ of trials.

In the experiments on White's effect, the stimulus configuration was more complex, allowing for more possible strategies on the part of the animals. For example, on the WGW stimulus, the fixation point was adjacent to a black band, but, on the BGB side, the fixation point was adjacent to a white band (Fig. 1D). Might it be that the high luminance white flank near the fixation point on the BGB side biased the animal to saccade to that side? One piece of evidence against this possibility has already been described: when the gray patches were identical, the animal saccaded almost entirely to the WGW side. This result is consistent with White's effect but inconsistent with the use of the bright band on the BGB side. The same conclusion can be reached referring to Figures 4 and 5. In all cases when the luminance of the two gray patches was the same, very few saccades were made to the BGB side.

Another possible strategy is that the animals were biased toward the WGW side. A conceivable reason for this might be that the animals averaged over an area larger than the gray patch. On the WGW side, averaging the white flanks along with the gray patch would give a higher luminance than averaging the black flanks with the other gray patch on the BGB side. A bias to the WGW side based on coarse spatial averaging is probably not responsible for the data because the percentage of saccades to the BGB side increased when the luminance of the black collinear bands on the BGB side was decreased (Fig. 6B). This is opposite the prediction of coarse spatial averaging but consistent with all the previously described evidence that the animals did base their decisions on brightness.

\section{Comparisons with human perception and other animal studies}

The first experiment we conducted examined brightness discrimination, and we derived psychometric functions (Fig. 2) that are qualitatively similar to functions for humans. In both species, there is a similar increase in the increment needed to discriminate brightness as mean luminance increases. Moreover, a quantitative comparison can be made of the Weber fractions in the two species. In our experiments, the Weber fraction for animal PN was 0.11 , and, for monkey HN, it was 0.18 . Human discrimination thresholds have been measured across a wide range of luminance, stimulus duration, stimulus size, and other variables, but the macaque values are well within the normal variance in the human data. For example, Cornsweet and Pinsker (1965) obtained a value of 0.14 . Our results are also consistent with previous work in nonhuman primates. In the brightness discrimination experiments, we used mean luminance settings of 12.3, 25.2, 37.0, 50.8, 81.9 , and $123.7 \mathrm{~cd} / \mathrm{m}^{2}$. Crawford (1935) computed a Weber fraction of $\sim 0.1$, with mean luminances of $2.5,23$, and $176 \mathrm{~cd} / \mathrm{m}^{2}$, and Brooks (1966) computed $\sim 0.2$.

In brightness induction, the monkey psychophysical curves are also comparable with those from humans. Brightness induction in humans has a nonlinear dependence on the luminance of the background annuli. The annulus surrounding a disk can cover a wide range of luminance, but as long as it is darker than the disk, it has relatively little effect on the brightness of the disk. When the luminance of the disk is raised near to, and then crosses through, the luminance of the disk, there is a precipitous drop in the brightness of the disk (Heinemann, 1955). These same features are exhibited in the macaque data in Figure 3. Some human studies (Heinemann, 1955) show that, when the annulus luminance is well below the disk luminance, the brightness of the disk actually increases as annulus luminance increases (i.e., brightness assimilation rather than brightness contrast). We did not see such assimilation effects in our macaque data, but, at the disk and annulus luminances we used in the monkey experiments, assimilation is not seen with humans either. Our results are qualitatively consistent with a previous study of brightness induction that reported the perceptual effect in several primate species (Davis et al., 1965). Unfortunately, it is difficult to quantitatively compare the previous results with our data. Davis et al. had animals compare two Munsell chips on black and white pieces of Masonite. Results were presented as single lightness matching points (i.e., one datum per animal); there were no data curves equivalent to our Figure 3. More importantly, because the properties (e.g., reflectance) of the Masonite backgrounds were not specified, it is not possible to establish a correspondence with the luminances in our experiments.

There have been no previous monkey studies of White's effect, but this interesting illusion is clearly perceived by macaques. Just as in humans, it appears to be opposite in sign to brightness contrast: when a gray patch is surrounded predominantly by black rather than white, the gray looks darker rather than lighter. We found that the basic effect generalizes across different test patch luminance levels. Also as in humans, if the flanks are reduced in size so that their length matches that of the gray patches, White's effect disappears and, instead, brightness induction is perceived (Moulden and Kingdom, 1989).

\section{Brightness processing and the brain}

Human brain areas critical for brightness processing have not yet been identified, but animal studies suggest that visual cortex performs important computations on the thalamic input. Responses correlated with brightness, brightness induction, the Cornsweet illusion, and lightness constancy have been found in area V1 (Reid and Shapley, 1989; Rossi et al., 1996; MacEvoy et al., 1998; Rossi and Paradiso, 1999; Hung et al., 2001; Kinoshita and Komatsu, 2001; MacEvoy and Paradiso, 2001). These findings do not prove that brightness computations necessarily take place in V1, but relevant signals exist in V1 unlike earlier in the system (Rossi and Paradiso, 1999). The quantitative similarity of brightness perception in monkeys and humans suggests that, if V1 performs critical brightness computations in animals, it might play a similar role in humans.

It is also possible that additional processing beyond V1 takes place. As mentioned in Results, our data are consistent with the idea that White's effect is a form of brightness induction that obeys particular "higher" rules. There is considerable debate about the extent to which White's effect requires more than bottom-up filtering (Moulden and Kingdom, 1989; Anderson, 1997; Todorovic, 1997; Blakeslee and McCourt, 1999; Kelly and Grossberg, 2000; Ross and Pessoa, 2000; Howe, 2001). Although controversial, a variety of brightness phenomena have been reported to depend on "higher" processing, including the analysis of illumination, depth, transparency, and grouping (Knill and Kersten, 1991; Adelson, 1993; Schirillo and Shevell, 1993; Buckley et al., 1994; Taya et al., 1995; Anderson, 1997; Kingdom et al., 1997; Schirillo and Shevell, 1997; Todorovic, 1997; Wishart et al., 1997; Gilchrist et al., 1999; Purves et al., 1999; Paradiso, 2000). It is only speculation at this point, but it seems likely that not all of these 
factors are analyzed in primary visual cortex. For this reason, the similarity of human and macaque brightness perception may indicate that visual processing of brightness is comparable in the two species well beyond V1.

\section{REFERENCES}

Adelson EH (1993) Perceptual organization and the judgement of brightness. Science 262:2042-2044.

Anderson BL (1997) A theory of illusory lightness and transparency in monocular and binocular images: the role of contour junctions. Perception 26:419-453.

Ash P (1951) The sensory capacities of infrahuman mammals: vision, audition, gustation. Psychol Bull 48:289-326.

Blakeslee B, McCourt ME (1999) A multiscale spatial filtering account of the White effect, simultaneous brightness contrast and grating induction. Vision Res 39:4361-4377.

Brooks BA (1966) Neurophysiological correlates of brightness discrimination in the lateral geniculate nucleus of the squirrel monkey. Exp Brain Res 2:1-17.

Buckley D, Frisby JP, Freeman J (1994) Lightness perception can be affected by surface curvature from stereopsis. Perception 23:869-881.

Cornsweet TN, Pinsker HM (1965) Luminance discrimination of brief flashes under various conditions of adaptation. J Physiol (Lond) 176:294-310.

Crawford MP (1935) Brightness discrimination in the rhesus monkey. Genet Psychol Monogr 17:75-160.

Davis RT, Masters HG, Tjomsland J (1965) Perception by monkeys. I. Psychophysical judgments of brightness by human and subhuman SS. Percept Mot Skills 20:637-654.

Gilchrist A, Kossyfidis C, Bonato F, Agostini T, Cataliotti J, Li X, Spehar B, Annan V, Economou E (1999) An anchoring theory of lightness perception. Psychol Rev 106:795-834.

Heinemann E (1955) Simultaneous brightness induction as a function of inducing- and test-field luminances. J Exp Psychol 50:89-96.

Howe PD (2001) A comment on the Anderson (1997), the Todorovic (1997), and the Ross and Pessoa (2000) explanations of White's effect. Perception 30:1023-1026.

Hung CP, Ramsden BM, Chen LM, Roe AW (2001) Building surfaces from borders in Areas 17 and 18 of the cat. Vision Res 41:1389-1407.

Judge SJ, Richmond BJ, Chu FC (1980) Implantation of magnetic search coils for measurement of eye position: an improved method. Vision Res 20:535-538.

Kayama Y, Riso RR, Bartlett JR, Doty RW (1979) Luxotonic responses of units in macaque striate cortex. J Neurophysiol 42:1495-1517.

Kelly F, Grossberg S (2000) Neural dynamics of 3-D surface perception: figure-ground separation and lightness perception. Percept Psychophys 62:1596-1618

Kingdom FA, Blakeslee B, McCourt ME (1997) Brightness with and without perceived transparency: when does it make a difference? Perception 26:493-506.

Kinoshita M, Komatsu H (2001) Neural representation of the luminance and brightness of a uniform surface in the macaque primary visual cortex. J Neurophysiol 86:2559-2570.

Knill DC, Kersten D (1991) Apparent surface curvature affects lightness perception. Nature 351:228-230.

Komatsu H, Murakami I, Kinoshita M (1996) Surface representation in the visual system. Brain Res Cogn Brain Res 5:97-104.

MacEvoy SP, Paradiso MA (2001) Lightness constancy in primary visual cortex. Proc Natl Acad Sci USA 98:8827-8831.

MacEvoy SP, Kim W, Paradiso MA (1998) Integration of surface information in primary visual cortex. Nat Neurosci 1:616-620.

Moulden B, Kingdom F (1989) White's effect: a dual mechanism. Vision Res 29:1245-1259.

Paradiso MA (2000) Visual neuroscience: illuminating the dark corners. Curr Biol 10:R15-R18.

Purves D, Shimpi A, Lotto RB (1999) An empirical explanation of the Cornsweet effect. J Neurosci 19:8542-8551.

Reid RC, Shapley RM (1989) Non-local effects in the perception of brightness: psychophysics and neurophysiology. In: Seeing contour and colour (Kulikowski JJ, Dickinson CM, Murray IJ, eds), pp 324-333. Oxford: Pergamon.

Ross WD, Pessoa L (2000) Lightness from contrast: a selective integration model. Percept Psychophys 62:1160-1181.

Rossi AF, Paradiso MA (1999) Neural correlates of perceived brightness in the retina, lateral geniculate nucleus, and striate cortex. J Neurosci 19:6145-6156.

Rossi AF, Rittenhouse CD, Paradiso MA (1996) The representation of brightness in primary visual cortex. Science 273:1391-1398.

Schilder P, Pasik T, Pasik P (1971) Extrageniculostriate vision in the monkey. II. Demonstration of brightness discrimination. Brain Res 32:383-398.

Schirillo JA, Shevell SK (1993) Lightness and brightness judgments of coplanar retinally noncontiguous surfaces. J Opt Soc Am A 10:2442-2452.

Schirillo JA, Shevell SK (1997) An account of brightness in complex scenes based on inferred illumination. Perception 26:507-518.

Spehar B, Gilchrist A, Arend LE (1995) The critical role of relative luminance relations in White's effect and grating induction. Vision Res 35:2603-2614.

Squatrito S, Trotter Y, Poggio GF (1990) Influences of uniform and textured backgrounds on the impulse activity of neurons in area V1 of the alert monkey. Brain Res 536:261-270.

Taya R, Ehrenstein WH, Cavonius CR (1995) Varying the strength of the Munker-White effect by stereoscopic viewing. Perception 24:685-694.

Todorovic D (1997) Lightness and junctions. Perception 26:379-394.

Wishart KA, Frisby JP, Buckley D (1997) The role of 3-D surface slope in a lightness/brightness effect. Vision Res 37:467-473. 